\title{
Netze und Leistungselektronik
}

\author{
R. Schürhuber OVE
}

angenommen am 18. Oktober 2021, online publiziert am 11. November 2021

(C) The Author(s) 2021

Energie aus erneuerbaren Quellen - insbesondere Wind- und PVAnlagen - macht einen immer größeren Anteil im Energiesystem aus und verdrängt konventionelle Einspeisung aus dem Netzbetrieb. Dies führt zu einem hohen Anteil an umrichterbasierter Erzeugung. Verstärkt wird dieser Trend dadurch, dass zunehmend auch konventionelle Anlagen mit umrichtergespeisten Einheiten gebaut bzw. im Zuge von Erneuerungen auf umrichtergespeiste Einheiten umgerüstet werden (z. B. aktuelle Großwasserkraftprojekte in Österreich). Der vermehrte Einsatz von leistungselektronischen Netzregel- und Übertragungselementen - z. B. HVDC-Links, FACTS (Flexible AC Transmission Systems) - und der Aufbau von E-MobilityLadeinfrastruktur und Speicherlösungen erhöhen die Anzahl an Umrichtern in allen Netzebenen zusätzlich.

Durch das zum Teil grundlegende unterschiedliche Verhalten dieser leistungselektronischen Komponenten im Vergleich zu konventionellen Erzeugungseinheiten und Lasten ergibt sich eine Vielzahl von neuen Herausforderungen für den Netzbetrieb, welche es zu bewältigen gilt. Dies betrifft unter anderem folgende Themenkreise:

Stabilität in umrichterdominierten Netzen Umrichtergekoppelte Erzeugung und Last bieten eine neue Form der Flexibilität in der Netzführung. Dies wird vor allem durch die adaptive und hochdynamische Regelfähigkeit für Wirkleistung in Kombination mit Speichern sowie durch die vielfältigen Möglichkeiten der Blindleistungsbereitstellung durch Umrichter möglich. So können z. B. kritische Netzsituationen dezentral mit einer entsprechenden Blindleistungsregelung und auch Wirkleistungseinspeisung entschärft werden. Prinzipielle Regelstrategien und Vorgaben dazu sind bereits in den aktuellen TOR-Richtlinien und dem RfG (Requirements for Generators, EU-Verordnung 2016/631) für Einzelanlagen angeführt. Allerdings wird es in umrichterdominierten Netzen zukünftig notwendig sein, Interaktionen der Anlagen untereinander sowie mit dem Gesamtsystem zu analysieren, um einen sicheren und effizienten Netzbetrieb zu gewährleisten. Forschungsbedarf gibt es insbesondere auch bei kombinierter DC- und AC-Leistungsübertragung. Aufgrund der rasanten Entwicklung der Umrichtertechnologie im hohen Leistungsbereich sind zukünftig vermehrt DC-Verbindungen im Netz zu erwarten. In Kombination mit AC-Verbindungen aufgrund der gegenseitigen Beeinflussung stellen diese neue Herausforderungen an die Stabilitätsanalyse und damit auch für die praktische Realisierung dar.

Transiente Stabilität im umrichterdominierten Netz Die dynamischen Eigenschaften eines von Umrichtern dominierten Netzes werden zunehmend durch die implementierten Steuer-, Regel- und Schutzalgorithmen bestimmt. Diese weisen - im Gegensatz zum bekannten Verhalten der Synchronmaschine und ihrer Regler - eine sehr große Diversität auf. Die Untersuchung der transienten Stabilität und der Wechselwirkung zwischen unterschiedlichen Umrichtern kann mit den klassischen Werkzeugen und Methoden der Stabilitätsanalyse nicht mehr durchgeführt werden, sodass hier spezielle Simulationsmethoden zur Untersuchung anzuwenden sind. Die Umrichterstabilität hat Einfluss auf folgende Aspekte des Netzbetriebs: o Reglerstabilität (Klein-/ Großsignal)

o Interaktion mit Netzresonanzen

o Bereitstellung von Augenblicksleistung - Verringerung ROCOF (Rate of Change of Frequency, zeitliche Änderungsrate der Frequenz)

o Kurzschlussstrombeitrag

o Fault-Ride-Through-Verhalten

o Beherrschung einer Systemauftrennung bzw. eines Inselbetriebs

o Schwarzstartfähigkeit

Verifikation der Netzanschlussbedingungen und Onlineüberprüfung des Regelverhaltens von Umrichtern Die in den aktuellen technischen Richtlinien festgelegten Testmethoden zur Überprüfung der Einhaltung der Anschlussbedingungen wurden für konventionell betriebene Energiesysteme entwickelt. Die dabei zugrunde liegenden vereinfachten Modelle sind nicht in der Lage, das tatsächliche Verhalten der Umrichter ausreichend genau zu reproduzieren, da dieses stark von den implementierten Regelungen abhängt. Um das geforderte Betriebs- und Fault-Ride-Through-Verhalten verifizieren zu können und Reglerinstabilitäten, -oszillationen und andere ungewünschte Interaktionen auszuschließen, ist es daher notwendig, eine vom Hersteller unabhängige Typprüfung mit geeigneten Hardware-in-the-Loop-Systemen durchzuführen. Zusätzlich ergibt sich ein Forschungsbedarf hinsichtlich der benötigten Messdaten aus Wide Area Measurement Systems (WAMS) für eine adäquate Online-Überprüfung von umrichtergekoppelten Energiesystemen in Betrieb. Durch die Möglichkeit von Controller-in-the-Loop-Tests, in welchen Steuerungskarten realer Anlagen in eine realitätsnahe Umgebung integriert und kritische Funktionen überprüft werden können, kann auch ein Beitrag zur Thematik der OT-Security geleistet werden.

Anforderungen an Schutzeinrichtungen in umrichterdominierten Netzen Die Reaktion einer umrichtergekoppelten Anlage auf unterschiedliche Netzsituationen wird hauptsächlich über die implementierte Regelstrategie festgelegt. Diese wird auf Mikroprozessoren ausgeführt, wobei Regelreaktionen im Mikrosekundenbereich erreicht werden. Die Spannungen und Ströme während einer netzkritischen Situation, wie dem Spannungseinbruch infolge eines Kurzschlusses, sind nicht mehr durch einfache Modelle erfassbar. Sie erfordern vielmehr eine umfassende Modellbildung, welche den Umrichter inklusive dessen Regelung beinhaltet. Bei der Integration von Umrichtern in bestehende Schutzkonzepte sind daher an verschiedenen Stellen Anpassungen nötig, um weiterhin einen zuverlässigen Netz- und Anlagenschutz zu gewährleisten. Dies kann sowohl durch Anpassung des Schutzes als auch durch eine adäquate Regelung des Umrichters erreicht werden.

Kurzfassung eines Vortrags bei der OVE-Energietechnik-Tagung, 58. Fachtagung der Energietechnik-Branche, die am 6. und 7. Oktober 2021 in Linz stattfand.

Schürhuber, Robert, Institut für Elektrische Anlagen und Netze, TU Graz, Inffeldgasse 18/1, 8010 Graz, Österreich (E-Mail: robert.schuerhuber@tugraz.at) 
Auswirkungen auf die Spannungsqualität und Anforderungen an die Umrichter Der stabile Betrieb von Umrichtern hängt von der Kurzschlussleistung und von der frequenzabhängigen Netzimpedanz im Anschlusspunkt ab. Speziell in schwachen Netzen kann die Interaktion von Umrichtern und Netzresonanzstellen zu Instabilitäten, begleitet von hohen Oberschwingungsemissionen, führen. Konventionelle Simulationen können diese Effekte nicht vollständig nachbilden und korrekt vorhersagen, für realitätsnahe Untersuchungen sind daher neue Werkzeuge und Methoden zur Behandlung und Lösung dieser Probleme zu entwickeln.

Insgesamt stellt diese Umstellung in der Landschaft der elektrischen Energieversorgung die größte Neuausrichtung seit der Strommarktliberalisierung dar und es bedarf einer Bündelung der Kräfte der Erzeuger, der Netzbetreiber, der Industrie sowie der Forschungseinrichtungen, um unser Energiesystem entsprechend anzupassen und weiterhin eine zuverlässige, leistbare und vor allem ökologisch tragbare Energieversorgung zu gewährleisten!

Funding Note Open access funding provided by Graz University of Technology.
Hinweis des Verlags Der Verlag bleibt in Hinblick auf geografische Zuordnungen und Gebietsbezeichnungen in veröffentlichten Karten und Institutsadressen neutral.

Open Access Dieser Artikel wird unter der Creative Commons Namensnennung 4.0 International Lizenz veröffentlicht, welche die Nutzung, Vervielfältigung, Bearbeitung, Verbreitung und Wiedergabe in jeglichem Medium und Format erlaubt, sofern Sie den/die ursprünglichen Autor(en) und die Quelle ordnungsgemäß nennen, einen Link zur Creative Commons Lizenz beifügen und angeben, ob Änderungen vorgenommen wurden. Die in diesem Artikel enthaltenen Bilder und sonstiges Drittmaterial unterliegen ebenfalls der genannten Creative Commons Lizenz, sofern sich aus der Abbildungslegende nichts anderes ergibt. Sofern das betreffende Material nicht unter der genannten Creative Commons Lizenz steht und die betreffende Handlung nicht nach gesetzlichen Vorschriften erlaubt ist, ist für die oben aufgeführten Weiterverwendungen des Materials die Einwilligung des jeweiligen Rechteinhabers einzuholen. Weitere Details zur Lizenz entnehmen Sie bitte der Lizenzinformation auf http://creativecommons.org/licenses/by/4.0/deed.de 